INPLASY

PROTOCOL

To cite: Mao et al.

Effectiveness and Safety of

Acupoint Catgut Embedding

for the Treatment of Post-

stroke constipation: a

systematic review and meta-

analysis. Inplasy protocol

202220041. doi:

10.37766/inplasy2022.2.0041

Received: 13 February 2022

Published: 13 February 2022

Corresponding author:

Mao Guo

511467235@qq.com

Author Affiliation:

Affiliated Hospital of Hunan

Academy of Chinese Medicine.

Support: Youth Fund Project.

Review Stage at time of this submission: Preliminary searches.

Conflicts of interest:

None declared.

\section{Effectiveness and Safety of Acupoint Catgut Embedding for the Treatment of Post-stroke constipation: a systematic review and meta-analysis}

\author{
Mao, G1; Xie, L2; Wang, QY3; Xie, Y4.
}

Review question / Objective: Population: Patients with a diagnosis of post-stroke constipation. Intervention: Acupoint catgut embedding therapy is one of the more common traditional Chinese medicine therapies, which uses absorbable surgical sutures to produce lasting acupoint stimulation in the human body according to the acupuncture theory of Traditional Chinese medicine. Those who are unwilling to accept acupoint catgut-embedding therapy or are allergic to absorbable surgical sutures, as well as those with scar constitution, will be excluded from this study. Comparison: (1)acupoint catgut embedding therapy versus sham acupoint catgut embedding therapy; (2)acupoint catgut embedding therapy versus conventional therapy; (3)acupoint catgut embedding therapy versus no treatment. Outcome: To compare the weekly average complete spontaneous bowel movements (CSBMs). The weekly average spontaneous bowel movements (SBMs), Bristol stool form score (BSFS), the score of the patient assessment of constipation quality of life questionnaire (PAC-QOL) and the score of defecation difficulty before and after treatment. Study design: We will include randomised controlled trials (RCTs).

INPLASY registration number: This protocol was registered with the International Platform of Registered Systematic Review and Meta-Analysis Protocols (INPLASY) on 13 February 2022 and was last updated on 13 February 2022 (registration number INPLASY202220041).

\section{INTRODUCTION}

Review question / Objective: Population: Patients with a diagnosis of post-stroke constipation. Intervention: Acupoint catgut embedding therapy is one of the more common traditional Chinese medicine therapies, which uses absorbable surgical sutures to produce lasting acupoint stimulation in the human body according to the acupuncture theory of Traditional Chinese medicine. Those who are unwilling 
to accept acupoint catgut-embedding therapy or are allergic to absorbable surgical sutures, as well as those with scar constitution, will be excluded from this study. Comparison: (1)acupoint catgut embedding therapy versus sham acupoint catgut embedding therapy; (2)acupoint catgut embedding therapy versus conventional therapy; (3)acupoint catgut embedding therapy versus no treatment. Outcome: To compare the weekly average complete spontaneous bowel movements (CSBMs). The weekly average spontaneous bowel movements (SBMs), Bristol stool form score (BSFS), the score of the patient assessment of constipation quality of life questionnaire (PAC-QOL) and the score of defecation difficulty before and after treatment. Study design: We will include randomised controlled trials (RCTs).

Condition being studied: Constipation is one of the common complications after stroke, and about $30 \%$ to $60 \%$ of stroke patients have or have suffered from constipation.Patients due to constipation and excessive exertion of defecation, easy to increase abdominal pressure, resulting in increased blood pressure, intracranial pressure, may cause another stroke, or even induced cerebral hernia endanger the patient's life, after stroke to maintain unobstructed stool is of great significance to the prognosis of stroke patients.At present, acupoint catgut embedding method is effective in the treatment of functional constipation, but there is little clinical evidence of high quality for the treatment of constipation after stroke.

\section{METHODS}

Participant or population: Patients with a diagnosis of post-stroke constipation.

Intervention: Acupoint catgut embedding therapy is one of the more common traditional Chinese medicine therapies, which uses absorbable surgical sutures to produce lasting acupoint stimulation in the human body according to the acupuncture theory of Traditional Chinese medicine.Those who are unwilling to accept acupoint catgut-embedding therapy or are allergic to absorbable surgical sutures, as well as those with scar constitution, will be excluded from this study.

Comparator: (1)acupoint catgut embedding therapy versus sham acupoint catgut embedding therapy(2)acupoint catgut embedding therapy versus conventional therapy(3)acupoint catgut embedding therapy versus no treatment.

Study designs to be included: We will include randomised controlled trials (RCTs).

Eligibility criteria: (1)Meet the diagnostic criteria of post-stroke constipation;(2) The age ranged from 18 to $80 ;(3)$ The vital signs were stable after stroke;(4)Patients or their family members signed informed consent and volunteered to participate in this study.

Information sources: We will perform a comprehensive search using in eight electronic databases including PubMed, Cochrane Library/Cochrane Central Register of Controlled Trials, Web of Science, Embase, China National Knowledge Internet, Chinese Biomedical Literature Database, Wanfang, and VIP databases. Key words or free-text terms are the following: "post-stroke constipation", "acupoint catgut embedding" and "Randomized clinical trials". There are no restrictions of countries, population characteristics, language.

Main outcome(s): To compare the weekly average complete spontaneous bowel movements (CSBMs).

Additional outcome(s): The weekly average spontaneous bowel movements (SBMs), Bristol stool form score (BSFS), the score of the patient assessment of constipation quality of life questionnaire (PAC-QOL) and the score of defecation difficulty before and after treatment.

Quality assessment / Risk of bias analysis: The risk of bias will be assessed by two reviewers (Le Xie and Yao Xie) independently according to the Cochrane 
Risk of Bia Assessment Tool. If there is any disagreement, the two reviewers shall discuss or negotiate with the third reviewer (Guo Mao) to solve it.

Strategy of data synthesis: All statistical analyses will be performed by the Cochrane Collaboration Review Manager software (RevMan 5.3). Relative Risk Ratio (RR) or risk difference (RD) with $95 \%$ confidence intervals $(\mathrm{Cl})$ will be used for dichotomous outcomes, while Mean Difference (MD) or Standard Mean Difference (SMD) will be used for continuous outcomes. $P<0.05$ indicates statistical significance. The $Q$ and $I^{2}$ statistics will be used to test the heterogeneity of the included literature. According to the heterogeneity test results, different effect models are used for metaanalysis. If the results between groups show statistical homogeneity $(P>0.05$, $\left.I^{2} \leq 50 \%\right)$, the fixed-effects model will be used for meta-analysis. If there is heterogeneity in the results between groups $\left(P<0.05, I^{2}>50 \%\right)$, the random effects model will be used. If there is significant clinical or methodological heterogeneity between studies, subgroup or sensitivity analyses will be performed to explore the sources of heterogeneity.

Subgroup analysis: If there is a sufficient number of studies, subgroups analysis will be done regarding disease outcome.

Sensitivity analysis: By excluding the included literatures one by one, the sensitivity analysis of the outcome indicators with high heterogeneity was carried out.Through the transformation effect model, the sensitivity analysis of the outcome indicators with small heterogeneity was carried out to evaluate the impact of the included studies on the robustness of the final results. If the heterogeneity of subgroup analysis and sensitivity classification is still high, metaregression is used to further analyze the sources of heterogeneity. Funnel plot was used to analyze publication bias, and Stata 14 software was used to conduct Egger's test to quantitatively evaluate publication bias.
Country(ies) involved: China.

Keywords: Acupoint Catgut Embedding; Post-stroke constipation; Meta-analysis; Traditional Chinese Medicine.

Contributions of each author:

Author 1 - Mao Guo - The author drafted the manuscript.

Email: 511467235@qq.com

Author 2 - Xie Le - The author contributed to the development of the selection criteria, and the risk of bias assessment strategy.

Email: 1289398706@qq.com

Author 3 - Wang Qinyu - The author provided statistical expertise.

Email: 3362001718@qq.com

Author 4 - Xie Yao - The author read, provided feedback and approved the final manuscript.

Email: xieyaohing@163.com 